\title{
The most common diseases among Syrian and Palestinian refugees to Lebanon: infections versus PTSD and chronic stress related diseases
}

\section{J. Suvada 1,2, A. Mátel 2 , L. Durisova2 ${ }^{2}$, I. Beldjebel 1,2 J. Božik²}

Original Articles

${ }^{1}$ St. Charles Foucould Clinic, Lebanon, Beirut

${ }^{2}$ St. Elisabeth University of Health and Social Sciences, Bratislava, Slovakia

\section{Correspondence to:}

St. Elisabeth University of Health and Social Sciences, Bratislava, Slovakia Nám. 1. Mája č. 1, 81000 Bratislava, Slovakia

Submitted: 15.6.2016

Revised: 24.8 .2016

Accepted: 11.9.2016

\section{Reviewers:}

V. Kozon

Allgemeines Krankenhaus - Medizinischer Universitätscampus, Vienna, Austria

V. Namulanda

Catholic University of Eastern Africa, Nairobi, Kenya

\section{Keywords:}

Immigrants, Stress, Infections, PTSD.

CSWHI 2016; 7(3): 47-48; DOI 10.22359/cswhi_7_3_13 @ 2016 Clinical Social Work and Health Intervention

\section{Introduction}

Syrian and Palestinian immigrants and refugees have arrived in Lebanon from diverse social, economic, educational, cultural and religious backgrounds for a variety of reasons: to seek work, education, economic advantage; to flee war, political upheaval or persecution; to join families from which they have been separated. Many of them have arrived with inadequate resources and suffer social exclusion and inequality of healthcare access. The acute phase following immigration, particularly from the war zones is the time when health can be neglected. Many come from healthcare systems that differ from traditional Western medicine; often may involve traditional remedies; health prevention is not part of daily life due to several reasons among immigrating families.

\section{Patients and Methods}

In our work, we share observations from 5 years of a treated cohort of refugees; we will show differences between acute and chronic stress and infectious diseases among immigrants from Syria and Palestine to Lebanon; highlight risk factors for these conditions among different age groups as well.

Lifestyle factors (together with lack of public health, preventive medicine and 
access to free healthcare) predispose some to specific diseases. Hereditary factors are sometimes important too, as in sickle cell disease, thalassaemia and glucose-6-phosphate dehydrogenase deficiency. Other problems may include malnutrition, intestinal parasites (Enterobius, Trichuris, Strongyloides and Ascaris), filariasis, leishmaniasis, Hepatitis A, B and C, tuberculosis, low immunization rate, typhoid fever, yellow fever, malaria, trachoma, syphilis, dengue fever, HIV infection, diarrheal diseases, leprosy, etc.

\section{Results}

In an acute phase patients can suffer from hypertension, coronary disease, diabetes, depression, gastrointestinal problems (e.g. lactose intolerance, non-organic abdominal pain), carcinomas of cervix, breast, colon and mouth, but also from vitamin D deficiency, alcoholism and substance abuse, and also social pathology as social determinants of health.

The second phase of transition with acculturation and modification of social norms, attitudes, values, behaviors and diet, bringing changes not least in the use of healthcare services even if it is in a similar ethnic region as the Near East and the Middle East typically takes at least 5 to 8 years. Most youth and young adults become well integrated into the community and they adapt to the social environment in Lebanon but there is still remain daily or occasional stress from family (e.g. social insecurity, no money to pay for admission to the hospital; any option to diagnose and then to treat serious diagnoses among family members as cancer, failure of the kidneys, inherited diseases or mental diseases).

The third phase, 10 or more years after arrival, is typified by the resettled refugee suffering a variety of chronic conditions at least partly as a consequence of resettlement, but many have acculturated and others continue to live and often work in their own communities within a multicultural society. In our work we share observation from 5 years treated cohort of refugees and we will show differences between acute and chronic stress and infectious diseases among immigrants from Syria and Palestine to Lebanon and highlight risk factors for these conditions among different age groups as well. 